\title{
MILD MANIFOLDS AND A NON-STANDARD RIEMANN EXISTENCE THEOREM
}

\author{
YA'ACOV PETERZIL AND SERGEI STARCHENKO
}

\begin{abstract}
Let $\mathcal{R}$ be an o-minimal expansion of a real closed field $R$ and $K$ be the algebraic closure of $R$. In earlier papers we investigated the notions of $\mathcal{R}$-definable $K$-holomorphic maps, $K$-analytic manifolds and their $K$-analytic subsets. We call such a $K$-manifold mild if it eliminates quantifiers after endowing it with all $K$-analytic subsets. Examples are compact complex manifolds and non-singular algebraic curves over $K$.

We examine here basic properties of mild manifolds and prove that when a mild manifold $M$ is strongly minimal and not locally modular then $M$ is bi-holomorphic with a non-singular algebraic curve over $K$.
\end{abstract}

\section{INTRODUCTION}

For a complex-analytic manifold $M$, let $\mathcal{M}$ be the first-order structure whose underlying set is $M$ and basic relations are all complex-analytic subsets of Cartesian powers of $M$.

The following result is due to Zilber (see [17]).

Theorem 1.0.1. If $M$ is a compact complex-analytic manifold, then $\mathcal{M}$ has elimination of quantifiers and has finite Morley rank. If $\mathcal{M}$ is strongly minimal then it is a Zariski geometry (see [8] for a definition of Zariski geometries).

Let $\mathcal{R}$ be a fixed o-minimal expansion of a real closed field $R$, and let $K=$ $R(\sqrt{-1})$ be its algebraic closure identified naturally with $R^{2}$. In a series of papers [12-14] we investigated notions $K$-holomorphic maps and $K$-analytic manifolds definable in the structure $\mathcal{R}$. For a $K$-analytic manifold $M$ let $\mathcal{A}(M)$ be the first order structure whose underlying set is $M$ and basic relations are all $K$-analytic subsets of Cartesian powers of $M$ (see Section 2.2 for definitions of $K$-analytic manifolds and $K$-analytic subsets). In paper [14] we showed that a version of the above Zilber's theorem holds in this general setting.

Date: January 3, 2008.

2000 Mathematics Subject Classification. Primary 03C64, 03H05, 32P05; Secondary 03C98, 30F99, 32C 25 .

Key words and phrases. O-minimality, Non-Archimedean complex analysis, Zariski geometries, Riemann existence theorem.

The second author was partially supported by the NSF. 
Theorem 1.0.2 ([14, Theorem 8.3]). qq If $M$ is a definably compact $K$-manifold, then $\mathcal{A}(M)$ has elimination of quantifiers and has finite Morley rank. If $\mathcal{A}(M)$ is strongly minimal then it is a Zariski geometry.

If we take $\mathcal{R}$ to be any o-minimal expansion of $\mathbb{R}_{\mathrm{an}}$, then every compact complex manifold can be viewed as an $\mathcal{R}$-definable object, and the structures $\mathcal{M}$ and $\mathcal{A}(M)$ are the same (see [11] for more details). Thus Theorem 1.0.2 can be considered as a proper generalization of Zilber's theorem.

Notice that the assumption of compactness is essential in Zilber's theorem. For example, if we take $M=\mathbb{C}$ then the structure $\mathcal{M}$ is not even stable (since the graph of complex exponential function is a complex-analytic subset of $\mathbb{C}^{2}$, we can use it to define integers in this structure.) However if we take $\mathcal{R}$ to be any ominimal expansion of the field of real numbers, then only complex analytic subsets of $\mathbb{C}^{n}$ definable in the structure $\mathcal{R}$ are algebraic (see [14, Theorem 5.1]), and we see a big difference between $\mathcal{M}$ and $\mathcal{A}(M)$ in this case. The structure $\mathcal{M}$ is unstable, and the structure $\mathcal{A}(M)$ is strongly minimal and inerdefinable with the field of complex numbers.

Motivated by this and other examples we define a $K$-analytic manifold $M$ to be mild if the structure $\mathcal{A}(M)$ has quantifier elimination. In particular, every definably compact $K$-manifold is mild, but there are also many mild manifolds that are not definably compact, e.g. any non-singular algebraic affine variety over $K$.

Our main goal is this paper is to prove the following theorem (see Theorem 6.0.5 below).

Theorem 1.0.3. gg2 Let $M$ be a strongly minimal mild $K$-manifold (i.e. $M$ is mild and the structure $\mathcal{A}(M)$ is strongly minimal). Then either the structure $\mathcal{A}(M)$ is locally modular or $M$ is $K$-biholomorphic with an algebraic curve over $K$.

One of obstacles in proving the above theorem is that in general Riemann existence theorem does not hold for mild manifolds: ${ }^{1}$ In paper [15] we considered a family of generalized 1-dimensional tori $\mathcal{E}_{\tau}$ and showed that for a sufficiently general nonstandard $\tilde{\tau}$ the structure $\mathcal{A}\left(\mathcal{E}_{\tilde{\tau}}\right)$ is strongly minimal but $\mathcal{E}_{\tilde{\tau}}$ is not $K$ biholomorphic to an algebraic curve. Thus, by the above theorem, $\mathcal{A}\left(\mathcal{E}_{\tilde{\tau}}\right)$ is locally modular.

To overcome this obstacle we prove in this paper that the second part of Riemann existence theorem holds for o-minimal expansions of real closed fields. Namely, every definable finite covering of a co-finite subset of $K$ is definably isomorphic to an algebraic covering (see Theorem 5.1.3 below). In a semialgebraic category this theorem can be restated as follows.

Theorem 1.0.4. Let $R$ be a real closed field and $K=R(\sqrt{-1})$ be its algebraic closure identified with $R^{2}$. Let $B \subseteq R^{2}$ be a co-finite subset, $C \subseteq R^{m}$ an $R$ semialgebraic set, and $\pi: C \rightarrow B$ an $R$-semialgebraic finite covering. Then there

\footnotetext{
${ }^{1}$ Due to a theorem of R. Moosa (see [10]) Riemann existence theorem holds in elementary extensions of $\mathcal{M}$ for a compact complex manifold $M$.
} 
is a nonsingular algebraic affine curve $\Gamma$ over $K$, a regular morphism $\tau: \Gamma \rightarrow K$, and an R-semialgebraic homeomorphism $f: C \rightarrow \Gamma$ such that $\pi=\tau \circ f$.

1.1. The structure of the paper. In Section 2 we review $K$-manifolds and $K$-analytic subsets.

In Section 3 we consider the field of $K$-meromorphic functions $\operatorname{Mer}(M)$ on a $K$-manifold $M$, and establish a connection between finite coverings and field extensions.

In Section 4 we define mild manifolds. The main result of this section is Theorem 4.3.3 stating that if $M$ is a strongly minimal mild manifold that is not locally modular then there is a nonconstant $K$-meromorphic function $f: M \rightarrow K$. It implies that, up-to finite subsets, every non locally modular strongly minimal mild manifold is a finite covering of $K$.

In Section 5 we show that the second part of Riemann existence theorem holds in o-minimal expansions of real closed fields. Since proofs of basic properties of definable finite coverings are analogous to classical proofs we present them in Appendix.

In Section 6 we prove Theorem 6.0.5

\section{Preliminaries}

For the rest of this paper we fix an o-minimal expansion $\mathcal{R}$ of a reald closed field $R$, and will denote by $K$ its algebraic closure $R(\sqrt{-1})$.

We briefly review the basic notions of analytic geometry with respect to the field $K$ and refer to [12-14] for more details.

2.1. $K$-holomorphic maps. The theory is based on the following straightforward analogue to the classical definition of a holomorphic map: Given a definable open set $U \subseteq K$ we call a definable function $f: U \rightarrow K K$-holomorphic if for every $z \in U$ the limit

$$
\lim _{z \rightarrow z_{0}} \frac{f(z)-f\left(z_{0}\right)}{z-z_{0}}
$$

exists in $K$ (we identify $K$ with $R^{2}$ in an obvious manner just like the complex field is identified with $\mathbb{R}^{2}$ ).

A definable map from an definable open subset $U \subseteq K^{n}$ into $K^{m}$ is called $K$ holomorphic if each of its coordinate functions is continuous and $K$-holomorphic in each variable separately.

Notice that in the classical setting, i.e. when $R$ is the field of real numbers, the above definitions amount to saying that the function $f$ is definable in the underlying o-minimal structure and is complex-holomorphic in the classical sense.

Let $U \subseteq K^{n}$ is a definable open set, $f: U \rightarrow K^{m}$ a $K$-holomorphic map, and $a \in U$. The $K$-differential $d f_{a}$ of $f$ at $a$ is a $K$-linear map from $K^{n}$ into $K$. We denote its rank by $\operatorname{Rnk}_{K}(f)_{a}$ and call it the $K$-rank of $f$ at $a$ (or just a rank of $f$ at $a$ if no confusion arises). 
2.2. $K$-manifolds. kman A definable $n$-dimensional $K$-manifold is a definable set $M$, equipped with a finite cover of definable sets $M=\bigcup_{i} U_{i}$, each of which is in definable bijection with an open subset of $K^{n}$ such that the transition maps are $K$-holomorphic maps between open subsets of $K^{n}$. As usual, $K$-holomorphic maps between $K$-manifolds are defined through the charts of the manifolds.

Let $M, N$ be $K$-manifolds, $U \subseteq M$ be a definable open subset, $f: U \rightarrow N$ a $K$-holomorphic map, and $a \in U$. The $K$-rank of $f$ at $a$ is defined using the charts. By the Chain Rule, this rank is independent of charts chosen.

Let $N$ be a $K$-manifold of $K$-dimension $n$. A definable subset $M \subseteq N$ is called $a$ d-dimensional $K$-submanifold if for every $a \in M$ there is a definable open set $U \subseteq N$ containing $a$ and a $K$-holomorphic $f: U \rightarrow K^{n-d}$ such that $M \cap U=f^{-1}(0)$ and $\operatorname{Rnk}_{K}(f)_{a}=n-d$. As was shown in [14, Lemma 3.3], every such submanifold admits a finite atlas, which makes it into a $K$-manifold on its own.

For example, if $X \subseteq K^{n}$ is a non-singular affine variety over $K$ then $X$ is a $K$-submanifold of $K^{n}$.

2.3. $K$-analytic sets. $A K$-analytic subset of a $K$-manifold $M$ is a definable set $A \subset M$, such that at every point $z \in M$, the set $A$ is given, locally near $z$, as the zero set of finitely many $K$-holomorphic functions. Again, in the classical setting a $\mathbb{C}$-manifold and a $\mathbb{C}$-analytic subset are just a complex manifold and a complex analytic subset, respectively, which are definable in the underlying ominimal structure over $\mathbb{R}$.

The $K$-dimension of a $K$-analytic set $A$ is defined to be the maximal $d$ such that $A$ contains a $d$-dimensional $K$-manifold. We use $\operatorname{dim}_{K} A$ to denote the dimension of $A$ as a $K$-analytic set and $\operatorname{dim}_{\mathcal{R}} A$ to denote its o-minimal dimension. We clearly have $\operatorname{dim}_{\mathcal{R}} A=2 \operatorname{dim}_{K} A$.

Let $M$ be a $K$-manifold, $A \subset M$ a $K$-analytic subset of $M$ and $X \subset A$. The set $X$ is a $K$-analytic subset of $A$ if it is $K$-analytic in $M$. The complement $A \backslash X$ of a $K$-analytic subset $X \subset A$ is called Zariski open in $A$.

Notice that, by Theorem 2.3.2 below, for an algebraic $K$-variety the above notion of a Zariski open subset coincides with the standard algebraic notion, so no confusion should arise.

If $A$ is a $K$-analytic subset of a $K$-manifold $M$ then we denote by $\operatorname{Reg}_{K}(A)$ the set of $K$-regular points of $A$, i.e. the set of all $a \in A$ such that $A$ is a $K$ submanifold of $M$ near $A$. As in the classical setting, the set $\operatorname{Reg}_{K}(A)$ is Zariski open and dense in $A$ (see[[14,6.1]]).

A $K$-analytic subset $A \subseteq M$ of a $K$-analytic manifold $M$ is called irreducible if it can not be written as $A=A_{1} \cup A_{2}$, where $A_{1}, A_{2}$ are $K$-analytic subset of $M$ and none of $A_{i}$ 's is contained in the other. If $A \subset M$ is irreducible $X \subset A$ a proper $K$-analytic subset of $A$ then $\operatorname{dim}_{K}(X)<\operatorname{dim}_{K}(A)$ and in particular the topological closure of $A \backslash X$ is $A$ ([[14, Corollary 4.10]]). We also have: 
Theorem 2.3.1 ([14, 4.6 and 4.12]). irred Every $K$-analytic subset $A$ of a $K$ manifold $M$ is a finite union of irreducible $K$-analytic subsets $A_{i}$ such that each $A_{i}$ is not contained in any other. (These are the irreducible components of $A$.) Moreover these irreducible components are exactly the closures of the definably connected components of $\operatorname{Reg}_{K}(A)$.

While the results so far are mostly analogues of the classical results, the theorems below are false without the o-minimality assumptions (see [14]).

The first result can be considered as a generalization of the classical Chow's lemma.

Theorem 2.3.2 ([14, 5.1]). Chow Let $A$ be a $K$-analytic subset of $K^{n}$ or of $\mathbb{P}^{n}(K)$. Then $A$ is an algebraic variety over $K$.

The following theorem on removal of singularities will be used here extensively:

Theorem 2.3.3 ([14, 4.13]). closure Let $M$ be a $K$-manifold and $E \subset M$ a $K$ analytic subset of $M$. If $A$ is a $K$-analytic subset of $M \backslash E$ then its topological closure in $M$ is a $K$-analytic subset of $M$.

The theorem below follows from Theorem 2.3.3 and the fact that $\operatorname{Reg}_{K}(A)$ is Zariski open in $A$.

Theorem 2.3.4. cap Let $A \subset M$ be an irreducible $K$-analytic subset of a $K$ manifold $M$. A set $X \subset \operatorname{Reg}_{K}(A)$ is a $K$-analytic subset of the $K$-manifold $\operatorname{Reg}_{K}(A)$ if and only if there is a $K$-analytic subset $Y$ of $M$ such that $X=$ $Y \cap \operatorname{Reg}_{K}(A)$.

Finally, as is shown in [14, Corollary 4.14], if $A$ is a $K$-analytic subset of a $K$-manifold then it remains $K$-analytic in elementary extensions of $\mathcal{R}$.

2.4. More on ranks of $K$-holomorphic maps. The following two theorems follow from properties of $\mathcal{R}$-differentiable maps in o-minimal structures (see for example [3, chapter7]).

Theorem 2.4.1. basicrank Let $f: M \rightarrow N$ be a $K$-holomorphic map between $K$-manifolds. Then

(1) If $r$ is the maximum rank of $f$ on $M$ then $\operatorname{dim}_{\mathcal{R}}(f(M))=2 r$ (we use $\mathcal{R}$-dimension for $f(M)$ because it is not a $K$-analytic set in general). Assume that $f$ is of constant rank $r$ on $M$. Then

(2) If $n=\operatorname{dim}_{K}(M)$ then for each $b \in f(M), f^{-1}(b)$ is a $K$-manifolds of dimension $n-r$;

(3) Each $a \in M$ has a definable open neighborhood $U$ such that $f(U)$ is a $K$-submanifold of $N$ of dimension $r$, In particular, if $\operatorname{dim}_{K}(N)=r$ then $f(M)$ is an open subset of $N$. 
It is easy to see that if $f: M \rightarrow N$ is a $K$-analytic map between $K$-manifolds and $k \in \mathbb{N}^{>0}$ then the set of all points $a \in M$ where the rank of $f$ is less than $k$ is $K$-analytic subset of $M$. It follows that $f$ has constant rank on a nonempty Zariski open subset of $M$.

2.5. Meromorphic maps. A $K$-meromorphic map from a $K$-manifold $M$ into a $K$-manifold $N$, is a definable $K$-holomorphic map $f$ from a Zariski open dense subset $\operatorname{dom}(f)$ of $M$ into $N$. As usual, we will identify two $K$-meromorphic maps $f, g$ from $M$ to $N$ if $f(x)=g(x)$ for all $x \in \operatorname{dom}(f) \cap \operatorname{dom}(g)$.

Remark 2.5.1. In paper [14], in the definition of a $K$-meromorphic map, we also required that the closure of the graph of $f$ in $M \times N$ is a $K$-analytic subset. However it was proved there (Corollary 9.1) that this extra assumption is not needed.

\section{THE FIELD OF MEROMORPHIC FUNCTIONS}

If $M$ is a definably connected $K$-manifold then it is not hard to see that the set of all $K$-meromorphic functions $\varphi: M \rightarrow K$ form a field under point-wise addition and multiplication. We will denote this field by $\operatorname{Mer}(M)$. Identifying $K$ with the constant functions on $M$ we will always consider $\operatorname{Mer}(M)$ as a field extension of $K$.

Let $f: M \rightarrow N$ be a $K$-meromorphic map between definably connected $K$ manifolds such that the range of $f$ is dense in $N$. It is not hard to see that for any $K$-meromorphic function $\varphi: N \rightarrow K$ the function $\varphi \circ f$ is a $K$-meromorphic function on $M$. Thus we have the map $\varphi \mapsto \varphi \circ f$ from $\operatorname{Mer}(N)$ to $\operatorname{Mer}(M)$. We will denote this map by $f^{*}$. Obviously, $f^{*}: \operatorname{Mer}(N) \rightarrow \operatorname{Mer}(M)$ is a field embedding.

3.1. K-meromorphic finite coverings. Recall that a continuous map between topological spaces $f: X \rightarrow Y$ is called an $n$-covering map, where $n \in \mathbb{N}^{>0}$, if every $b \in Y$ has an open neighborhood $V$ such that $f^{-1}(V)$ consists of $n$ pairwise disjoint sets $U_{1}, \ldots, U_{n}$ and $f$ maps each $U_{i}$ homeomorphically onto $V$. We call it a finite covering map if it is an $n$-covering map for some $n$. The following is immediate.

Claim 3.1.1. ncov A continuous map between Hausdorff topological spaces $f: X \rightarrow$ $Y$ is an $n$-covering if and only if $f$ is a local homeomorphism and $\left|f^{-1}(b)\right|=n$ for any $b \in Y$.

Definition 3.1.2. A $K$-meromorphic map $f: M \rightarrow N$ between $K$-manifolds is $a$ $K$-meromorphic $n$-covering map if there is a Zariski open dense subset $U$ of $M$ and a Zariski open dense subset $V$ of $N$ such that that $U \subset \operatorname{dom}(f)$ and $f\lceil U: U \rightarrow V$ is an $n$-covering.

It is called a $K$-meromorphic finite covering if it is a $K$-meromorphic $n$-covering map for some $n \in \mathbb{N}>0$.

Remark 3.1.3. It is not hard to see that if $f: M \rightarrow N$ is a $K$-meromorphic finite covering then $M$ and $N$ have the same dimension. 
Theorem 3.1.4. cover Let $M, N$ be definably connected $K$-manifolds. If $f: M \rightarrow$ $N$ is a K-meromorphic $n$-covering then $\operatorname{Mer}(M)$ is a finite extension of $f^{*}(\operatorname{Mer}(N))$ of degree at most $n$.

Proof. By the Primitive Element Theorem, it is sufficient to show that for any $\alpha(x) \in \operatorname{Mer}(M)$ there are $c_{1}(y), \ldots, c_{n}(y) \in \operatorname{Mer}(N)$ and a Zariski open dense $U \subseteq M$ such that

$$
\alpha^{n}(x)+c_{1}(f(x)) \alpha^{n-1}(x)+\cdots+c_{n-1}(f(x)) \alpha(x)+c_{n}(f(x))=0
$$

for all $x \in U$. Obviously, we need to consider only non-constant $\alpha$.

Let $U \subseteq M$ and $V \subseteq N$ be Zariski open dense subsets such that $f: U \rightarrow V$ is an $n$-covering. Decreasing $U$ and $V$ if needed we can assume that $\alpha(x)$ is $K$ holomorphic on $U$.

Using definable choice we can find definable $\xi_{1}, \ldots, \xi_{n}: V \rightarrow U$ such that $f^{-1}(y)=\left\{\xi_{1}(y), \ldots, \xi_{n}(y)\right\}$ for all $y \in V$.

For $i=1, \ldots, n$, let $s_{i}\left(t_{1}, \ldots, t_{n}\right)$ be the $i$-th basic symmetric polynomial in $n$ variables.

Consider functions $c_{i}$ from $V$ into $K$, defined as

$$
c_{i}(y)=(-1)^{i} s_{i}\left(\alpha\left(\xi_{1}(y)\right), \ldots, \alpha\left(\xi_{n}(y)\right)\right) .
$$

Using the symmetry of $s_{i}$ 's, it is not difficult to see that each $c_{i}$ is $K$-holomorphic on $V$ (see [13, bottom of p.16] for a similar argument).

For $x \in U$ and $y=f(x)$ we have $\prod_{i=1}^{n}\left(x-\alpha\left(\xi_{i}(y)\right)\right)=0$.

Thus

$$
\alpha^{n}(x)+c_{1}(f(x)) \alpha^{n-1}(x)+\cdots+c_{n-1}(f(x)) \alpha(x)+c_{n}(f(x))=0
$$

on $U$.

\section{The Structure $\mathcal{A}(M)$ AND Mild MANifolds}

Let $M$ be a $K$-manifold. We denote by $\mathcal{A}(M)$ the first order structure whose universe is $M$ and basic relations are $K$-analytic subsets of Cartesian powers of $M$.

Remark 4.0.5. rem.mild The structure $\mathcal{A}(M)$ depends, by definition, on the ambient o-minimal structure $\mathcal{R}$ (because the definition of a $K$-analytic set depends on that structure).

Let $\mathcal{R}^{*}$ be an $|R|^{+}$-saturated elementary extension of $\mathcal{R}$. We denote by $R^{*}$ the universe of $\mathcal{R}^{*}$, by $K^{*}$ its algebraic closure and for an $\mathcal{R}$-definable subset $X \subseteq R^{n}$ we denote by $X^{*}$ the set of its $\mathcal{R}^{*}$-points. It is easy to see that $M^{*}$ is a $K^{*}$-manifold, and as was pointed out earlier, if $A$ is a $K$-analytic subset of $M$ then $A^{*}$ is $K^{*}$-analytic subset of $M^{*}$. We let $\mathcal{A}\left(M^{*}\right)$ denote the first order structure whose universe is $M^{*}$ and basic sets are $K^{*}$-analytic subsets of Cartesian powers of $M^{*}$.

It is easy to see that there is a reduct $\widetilde{M}$ of $\mathcal{A}\left(M^{*}\right)$ such that $\widetilde{M}$ is an $|M|^{+}$-saturated elementary extension of $\mathcal{A}(M)$. However, as is shown in [13], the 
structures $\mathcal{A}\left(M^{*}\right)$ and $\widetilde{M}$ may differ, in terms of their definable sets. For more on the relationship between $\mathcal{A}\left(M^{*}\right)$ and $\widetilde{M}$, in the case where $\mathcal{R}=\mathbb{R}_{a n}$, see [11].

4.1. Mild manifolds. Our notion of a mild manifold was inspired by Zilber's observation that compact complex manifolds with their analytic structure, eliminate quantifiers (see [20]).

Definition 4.1.1. We say that a $K$-manifold $M$ is mild if the structure $\mathcal{A}(M)$ admits quantifier elimination.

If $\mathcal{A}(M)$ is mild then every definable $X \subset M^{k}$ can be written as $X=$ $\bigcup_{i=1}^{k}\left(A_{i} \backslash B_{i}\right)$, where $A_{i} \subset B_{i}$ are $K$-analytic subsets of $M^{k}$ and each $A_{i}$ is irreducible. It follows that the topological closure of $X$ is the union of the $A_{i}$ 's and therefore $K$-analytic itself. It is not difficult now to conclude:

Theorem 4.1.2. faith Let $M$ be a mild $K$-manifold, $A \subset M^{n}$ a $K$-analytic irreducible subset, and $\pi: M^{n} \rightarrow M^{k}$ a projection. Then the topological closure $\operatorname{cl}(\pi(A))$ of $\pi(A)$ is an irreducible $K$-analytic subset of $M^{k}$ and $\operatorname{cl}(\pi(A)) \backslash F \subset \pi(A)$ for some $K$-analytic subset $F \varsubsetneqq \operatorname{cl}(\pi(A))$.

The example above together with the theorem below provide us with a wide class of mild $K$-manifolds.

Theorem 4.1.3. reqe Let $M$ be a mild $K$-manifold and $A \subset M$ a $K$-analytic subset of $M$. Then

(i) $M \backslash A$ is a mild manifold.

(ii) If $A$ is irreducible then $\operatorname{Reg}_{K}(A)$ is a mild $K$-manifold.

Proof. (i) Follows from Theorem 2.3.3, while (ii) follows from Theorem 2.3.4.

Example 4.1.4. As the theorem below shows, the unit disk $D \subseteq K$ is not a mild $K$-manifold, in any o-minimal structure over $R$.

Claim 4.1.5. faithk For a definable open nonempty subset $U \subseteq K$ the following conditions are equivalent.

(1) $U$ is co-finite in $K$.

(2) $U$ is a mild $K$-manifold.

(3) The structure $\mathcal{A}(U)$ is strongly minimal.

Proof. (1) $\Rightarrow(2)$. If $U \subset K$ is co-finite then, by Theorems 2.3.2 and 2.3.3, the structure $\mathcal{A}(U)$ is the same as the structure induced on $U$ by algebraic sets, hence it is strongly minimal.

(2) $\Rightarrow(3)$. Since $\operatorname{dim}_{K}(U)=1$ all proper $K$-analytic subsets of $U$ are finite. By quantifier elimination, every $\mathcal{A}(U)$-definable subset of $U$ is either finite or co-finite. Since o-minimal structures have uniform finiteness property, the structure $\mathcal{A}(U)$ is strongly minimal.

$(3) \Rightarrow(1)$. Assume now that $U$ is not co-finite in $K$ and let $X=K \backslash U$. Obviously, for any $c \in K$ the them map $x \mapsto c+x$ is a bijection between $U$ and $c+U$ that 
induces bijection between $\mathcal{A}(U)$-definable sets and $\mathcal{A}(c+U)$ definable sets. Thus we may assume $0 \in U$. For every $a \in K$ the set $\{(x, y) \in U \times U: x=a y\}$ is a $K$-analytic subset of $U \times U$, hence the set $a U \cap U$ is definable in $\mathcal{A}(U)$. If we take $a$ close enough to 0 so that $a X \cap U$ is infinite, we obtain that $a U \cap U$ is infinite and co-infinite. Hence $\mathcal{A}(U)$ is not strongly minimal.

We need the following technical result.

Lemma 4.1.6. milder Let $M$ be a mild $K$-manifold, $A$ an irreducible $K$-analytic subset of $M^{n}, \pi: M^{n} \rightarrow M^{k}$ a projection such that $\pi(A)$ is infinite, and let $B$ be the topological closure of $\pi(A)$ in $M^{k}$. Then there is a Zariski open dense subset $U$ of $\operatorname{Reg}_{K}(A)$ such that $\pi$ has a constant rank on $U$ and $\pi(U)$ is a Zariski open dense subset of $\operatorname{Reg}_{K}(B)$.

Proof. Let $r$ be the maximal rank of $f$, restricted to the manifold $\operatorname{Reg}_{K}(A)$. As was pointed out earlier, there exists a Zariski open dense subset $U_{0} \subseteq \operatorname{Reg}_{K}(A)$ such that $\pi$ has constant rank $r$ on $U_{0}$. The set $\pi\left(U_{0}\right)$ is dense in $B$ hence, by quantifier elimination, $\pi\left(U_{0}\right)$ contains a Zariski open dense subset $W$ of $B$. We can now take $U=\pi^{-1}\left(W \cap \operatorname{Reg}_{K}(B)\right)$.

4.1.1. Some open questions on mild manifolds. Claim 4.1.5 suggests an immediate question:

Question. Is it true that a definable open $U \subset K^{n}$ is mild iff it is Zariski open in $K^{n}$ ?

Question. Let $M$ be a $K$-manifold. Assume that $\mathcal{A}(M)$ is strongly minimal, or even stable. Is $M$ necessarily mild?

Question. Let $M, N$ be mild $K$-manifolds. Is $M \times N$ necessarily mild?

Let $\mathcal{R}^{*} \succ \mathcal{R}, R^{*}, K^{*}$ and $\widetilde{M}$ and $\mathcal{A}\left(M^{*}\right)$ be as in Remark 4.0.5. Notice that if $\mathcal{A}(M)$ is mild then the structure $\widetilde{M}$ eliminates quantifiers. However, one can still ask:

Question Let $\mathcal{R}^{*}, M^{*}, \mathcal{A}\left(M^{*}\right)$ and $\widetilde{M}$ be as in Remark 4.0.5. Assume that $\mathcal{A}(M)$ is mild.

1. Is the structure $\mathcal{A}\left(M^{*}\right)$ mild as well?

2. Does $\mathcal{A}(M)$ remain mild in every o-minimal expansion of the structure $\mathcal{R}$ ?

4.2. Generic Types. Let $M$ be a mild $K$-manifold. If $A$ is an irreducible $K$ analytic subset of $M^{n}$ then, by quantifier elimination, in the structure $\mathcal{A}(M)$ the set $\{U \subset A: U$ is Zariski open and dense in $\mathrm{A}\}$ is contained in a unique complete $n$-type over $M$. As usual we will call this type a generic type of $A$. Conversely, if $p$ is a complete $n$-type over $M$ and $A \in p$ is an irreducible $K$-analytic subset of minimal $K$-dimension then $p$ is the generic type of $A$.

\subsection{Strongly minimal mild $K$-manifolds.}

Definition 4.3.1. A $K$-manifold $M$ is called a strongly minimal mild $K$-manifold if it is mild and the structure $\mathcal{A}(M)$ is strongly minimal. 
Since every o-minimal structure has uniform bounds on definable families of finite sets, it follows that a mild $K$-manifold $M$ is strongly minimal if and only if every proper $K$-analytic subset of $M$ is finite. In particular, every definably connected definably compact 1 -dimensional $K$-manifold is strongly minimal and mild.

Theorem 4.3.2. qq1 If $M$ is a strongly minimal mild manifold then the structure $\mathcal{A}(M)$ is a Zariski geometry with $K$-analytic subsets taken as closed ones.

Proof. Using the dimension theorem [14, Theorem 7.3], it is easy to see that strongly minimal mild manifolds with $K$-analytic subsets satisfy the axioms of Zariski geometries from [8].

Our main goal in this section is to prove the following theorem.

Theorem 4.3.3. mainmild Let $M$ be a mild strongly minimal $K$-manifold. The structure $\mathcal{A}(M)$ is locally modular if and only if $\operatorname{Mer}(M)=K$.

We will need several claims.

Claim 4.3.4. genm Let $M$ be a strongly minimal mild $K$-manifold. Then for any $n \in \mathbb{N}^{>0}$ the generic type of $M^{n}$ is the unique n-type in $\mathcal{A}(M)$ whose Morley rank is $n$.

Proof. Easy, by induction on $n$.

Claim 4.3.5. gena Let $M$ be a strongly minimal mild $K$-manifold and $A \subset M^{n}$ an irreducible $K$-analytic subset. Then there is a projection $\pi: M^{n} \rightarrow M^{k}$ such that $\pi\left\lceil\operatorname{Reg}_{K}(A): \operatorname{Reg}_{K}(A) \rightarrow M^{k}\right.$ is a K-meromorphic finite covering.

Proof. Let $p(\bar{x})$ be the generic type of $A$. Notice that the formula $x \in \operatorname{Reg}_{K}(A)$ belongs to $p$. Let $\bar{a}=\left(a_{1}, \ldots, a_{n}\right)$ be a realization of $p$ in some elementary extension $\widetilde{M}$ of $\mathcal{A}(M)$ (see discussion in Remark 4.0.5).

Let $k$ be the Morley rank of $p$. After permuting coordinates if needed, we may assume that in the structure $\widetilde{M}$, the elements $a_{1}, \ldots, a_{k}$ are algebraically independent over $M$ and $\bar{a}$ is in the algebraic closure of $a_{1}, \ldots, a_{k}$. Let $\pi: M^{n} \rightarrow$ $M^{k}$ be the projection on-to the first $k$-coordinates.

By Claim 4.3.4, $\left(a_{1}, \ldots, a_{k}\right)$ realizes the generic type of $M^{k}$. Thus there is $m \in \mathbb{N}^{>0}$ such that the set

$$
V_{0}=\left\{x \in M^{k}:\left|\pi^{-1}(x) \cap \operatorname{Reg}_{K}(A)\right|=m\right\}
$$

is Zariski open and dense in $M^{k}$.

Let $U_{0}=\pi^{-1}\left(V_{0}\right) \cap \operatorname{Reg}_{K}(A)$. Obviously it is a Zariski open subset of $A$. By Theorem 4.1.6 there is a Zariski open dense $U \subset U_{0}$ such that $\pi \uparrow U$ has constant rank and $V=\pi(U)$ is a Zariski open dense subset of $M^{k}$. It is easy to see that $\pi\lceil U: U \rightarrow V$ is a $K$-holomorphic $m$-covering, and therefore $\pi \mid \operatorname{Reg}_{K}(A): \operatorname{Reg}_{K}(A) \rightarrow M^{k}$ is a $K$-meromorphic finite covering.

Claim 4.3.6. kmerom Let $M$ be a strongly minimal mild $K$-manifold and $f: M \rightarrow$ $K$ a non-constant $K$-meromorphic function. Then $f: M \rightarrow K$ is a $K$-meromorphic finite covering. In particular $\operatorname{dim}_{K}(M)=1$. 
Proof. Let $U \subset M$ be a Zariski open set on which $f$ is $K$-holomorphic. By the strong minimality of $\mathcal{A}(M)$, the set $M \backslash U$ is finite. Because $f$ is nonconstant, the set $f(U)$ is infinite. It follows that the set $\{u \in U: d f(u)=0\}$ is a proper $K$-analytic subset of $U$ which, by 2.3.4, is also definable in $\mathcal{A}(M)$, hence must be co-finite. Shrinking $U$, if needed, we may assume that the differential of $f$ is nowhere zero on $U$.

Consider the equivalence relation $E$ on $U$ defined as $x E y \Longleftrightarrow f(x)=f(y)$. Clearly, $E$ is a $K$-analytic subset of $U \times U$ and, by Theorem 2.3.3, it is definable in $\mathcal{A}(M)$. By strong minimality, almost all $E$-classes have the same (finite) size. Shrinking $U$ again, if needed, we may assume that all $E$-classes have the same size $n$. In particular, we already see that $\operatorname{dim}_{K} M=\operatorname{dim}_{K} K=1$, and by our assumption on $d f$, the map $f: U \rightarrow f(U)$ is a $n$-covering map. We are left to show that $f(U)$ is Zariski open and dense in $K$, i.e. is co-finite in $K$.

Since for any $K$-analytic subset $A \subseteq f(U)$ the closure of $f^{-1}(A)$ in $M$ is $K$-analytic in $M$ we obtain that the structure $\mathcal{A}(f(U))$ is isomorphic to a reduct of the structure on $U / E$ induced by $\mathcal{A}(M)$. Since $\mathcal{A}(M)$ is strongly minimal, it implies that $\mathcal{A}(f(U))$ is strongly minimal as well, and, by the Claim 4.1.5, $f(U)$ is co-finite in $K$.

As a corollary we obtain one of the directions in Theorem 4.3.3.

Corollary 4.3.7. notlm Let $M$ be a mild strongly minimal $K$-manifold. If $\operatorname{Mer}(M) \neq$ $K$ then the structure $\mathcal{A}(M)$ is not locally modular.

Proof. If $\operatorname{Mer}(M) \neq K$ then, by the previous claim, there is a $K$-meromorphic finite covering $f: M \rightarrow K$. It is easy to see that the field $K$ is interpretable in $\mathcal{A}(M)$, hence $\mathcal{A}(M)$ is not locally modular.

4.3.1. Proof of Theorem 4.3.3. In this section we prove the second direction of Theorem 4.3.3. We fix a strongly minimal mild $K$-manifold $M$ with $\operatorname{Mer}(M)=K$ and will show that $\mathcal{A}(M)$ is locally modular.

Claim 4.3.8. claim1

(1) For any $n \in \mathbb{N}^{>0}$ we have $\operatorname{Mer}\left(M^{n}\right)=K$

(2) For any irreducible $K$-analytic subset $A \subset M^{n}$, we have $\operatorname{Mer}\left(\operatorname{Reg}_{K}(A)\right)=K$.

Proof. (1) is easy.

(2) By Theorem 4.3.5 there is a projection $\pi: M^{n} \rightarrow M^{k}$ such that $\pi: \operatorname{Reg}_{K}(A) \rightarrow$ $M^{k}$ is a $K$-meromorphic finite covering. Applying Theorem 3.1.4 we obtain that $\operatorname{Mer}\left(\operatorname{Reg}_{K}(A)\right)$ is a finite extension of $\operatorname{Mer}\left(M^{k}\right)=K$. Since $K$ is algebraically closed, it does not have proper finite extensions, hence $\operatorname{Mer}\left(\operatorname{Reg}_{K}(A)\right)=K$.

Towards getting a contradiction we assume that $\mathcal{A}(M)$ is not locally modular.

Remark 4.3.9. The work [8] of Hrushovski and Zilber on Zariski structures implies that if $M$ is not locally modular then it is a finite cover of an algebraic curve 
over an $\mathcal{A}(M)$-definable field. However, because we need a more direct relationship to our underlying field $K$ we take a different, more analytic, approach. The method we are using below is based on the techniques of Camapana and Fujiki in the setting of compact complex manifolds, as interpreted in [18] by Pillay and Ziegler and, in the o-minimal setting, in [14].

Let $\mathcal{R}^{*} \succ \mathcal{R}$ be an $|R|^{+}$-saturated elementary extension of $\mathcal{R}$. We let $R^{*}, K^{*}$, $\mathcal{A}\left(M^{*}\right)$ and $\widetilde{M} \succ \mathcal{A}(M)$ be as in Remark 4.0.5. Notice that since every $m \in M$ is a $K$-analytic subset of $M$, we have that $M=\operatorname{acl}(\emptyset)$ in $\widetilde{M}$.

Since $\mathcal{A}(M)$ is not locally modular and $\widetilde{M}$ is sufficiently saturated, there are $a \in\left(M^{*}\right)^{2}, b_{i} \in\left(M^{*}\right)^{l}, i \in \omega$, and an $\mathcal{A}(M)$-formula $\theta(x, y)$ such that in the structure $\widetilde{M}$ :

(1) $\operatorname{tp}\left(a b_{i}\right)=\operatorname{tp}\left(a b_{j}\right)$ for all $i, j \in \omega$;

(2) $\operatorname{tp}\left(a / b_{i}\right)$ is strongly minimal for each $i \in \omega$;

(3) $\widetilde{M} \models \theta\left(a, b_{i}\right)$ for all $i \in \omega$;

(4) $\theta\left(x, b_{i}\right) \wedge \theta\left(x, b_{j}\right)$ is finite for all $i \neq j \in \omega$.

Let $C \subset M^{2+l}$ be the irreducible $K$-analytic subset such that $\operatorname{tp}\left(a b_{0}\right)$ is the generic type of $C$. By quantifier elimination we can assume that $\theta(x, y)$ defines a Zariski open dense subset $W_{0}$ of $C$. We will denote by $\pi_{2}$ and $\pi_{l}$ the projections $\pi_{2}: M^{2+l} \rightarrow M^{2}$ and $\pi_{l}: M^{2+l} \rightarrow M^{l}$. Let $A$ be the topological closure of $\pi_{2}(C)$ in $M^{2}$ and let $B$ be the topological closure of of $\pi_{l}(C)$ in $M^{l}$. By Theorem 4.1.2, $A$ is an irreducible $K$-analytic subset of $M^{2}$ and $B$ is an irreducible $K$-analytic subset of $M^{l}$.

Applying Theorem 4.1.6 we can find a Zariski open dense subset $W \subset W_{0} \cap$ $\operatorname{Reg}_{K}(C)$ such that:

(a) both $\pi_{2}$ and $\pi_{l}$ have constant rank on $W$;

(b) the set $U=\pi_{2}(W)$ is a $K$-submanifold of $M^{2}$ and Zariski open in $A$;

(c) the $V=\pi_{l}(W)$ is a $K$-submanifold of $M^{l}$ and Zariski open in $B$.

We will consider $W$ as a $K$-submanifold of $U \times V$.

We now work in the structure $\mathcal{R}^{*}$. For $y \in V^{*}$ let

$$
U_{y}^{*}=\left\{x \in U^{*}:(x, y) \in W^{*}\right\} .
$$

Applying Theorem 9.1 from[16 $]^{2}$ we obtain that there is a $K^{*}$-holomorphic vector bundle $\tau: L \rightarrow U^{*}$ and $K^{*}$-holomorphic map $\mu: W^{*} \rightarrow \mathbb{P}(L)$ such that the following diagram is commutative:

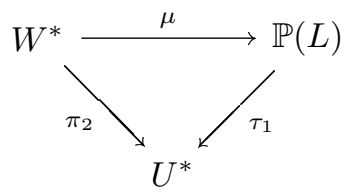

\footnotetext{
${ }^{2}$ Theorem 9.1 and its proof in [16] were formulated in the context of the real field, however the proof was written with the nonstandard setting in mind and carries over to our setting
} 
and $\mu(x, y)=\mu\left(x, y^{\prime}\right)$ if and only if $U_{y}^{*}=U_{y^{\prime}}^{*}$ near $x$.

Since the type $\operatorname{tp}\left(a b_{0}\right)$ is the generic type of $C$ we obtain that $\left(a b_{i}\right) \in W^{*}$ for all $i \in \omega$, and, since $U_{b_{i}}^{*} \cap U_{b_{j}}^{*}$ is finite for $i \neq j$, we have that $\mu\left(a, b_{i}\right) \neq \mu\left(a, b_{j}\right)$ for $i \neq j$.

Let $V_{a}^{*}=\left\{y \in V^{*}:(a, y) \in W^{*}\right\}$. Notice that all $b_{i}$ are in $V_{a}^{*}$. After fixing a basis for $\tau^{-1}(a)$ we can identify $\tau_{1}^{-1}(a)$ with a projective space $\mathbb{P}_{d}\left(K^{*}\right)$. Let $f: V_{a}^{*} \rightarrow \mathbb{P}_{d}\left(K^{*}\right)$ be the map $y \mapsto \mu(a, y)$. Obviously $f$ is $K^{*}$-holomorphic, and, since $f\left(b_{i}\right) \neq f\left(b_{j}\right)$ for $i \neq j$, we have that the range of $f$ is infinite.

Since $\mathcal{R}^{*}$ is an elementary extension of $\mathcal{R}$, we can find $\alpha \in V$ and $K$ holomorphic map $g$ from $V_{\alpha}=\{y \in V:(\alpha, y) \in W\}$ into $\mathbb{P}_{d}(K)$ whose image is infinite. It is not hard now to derive a contradiction to Claim 4.3.8(2).

This finishes the proof of Theorem 4.3.3.

\section{ON THE NON-STANDARD RIEMANN EXISTENCE THEOREM}

Recall that the classical Riemann existence theorem states that every compact Riemann surface is bi-holomorphic with an algebraic curve. This theorem fails in the category of $K$-analytic manifolds. Namely, there is an example of a definably compact $K$-manifold of $K$-dimension one that is not $K$-biholomorphic with an algebraic curve (see [15, Corollary 5.6]).

In fact, Riemann existence theorem can be restated as having two parts.

Theorem 5.0.10 (Riemann existence theorem). ret Let $M$ be a compact connected Riemann surface.

(1) There exists a non-constant holomorphic map $\pi: M \rightarrow \mathbb{P}_{1}(\mathbb{C})$.

(2) For any such $\pi$ there is a non-singular algebraic curve $\Gamma$ over $\mathbb{C}$, a regular map $\tau: \Gamma \rightarrow \mathbb{P}_{1}(\mathbb{C})$ and a bi-holomorphic map $f: M \rightarrow \Gamma$ such that $\pi=$ $\tau \circ f$.

The second part of the above theorem can be restated slightly more generally.

Theorem 5.0.11. ret2 Let $B$ be a co-finite subset of $\mathbb{C}, X$ a topological manifold, and $\pi: X \rightarrow B$ a finite covering. Then there exist an affine non-singular algebraic curve $\Gamma$ over $\mathbb{C}$, a regular map $\tau: \Gamma \rightarrow B$ and a homeomorphism $f: X \rightarrow \Gamma$ such that $\pi=\tau \circ h$.

Remark 5.0.12. The above theorem is well-known, but since we could not find it anywhere in the literature, we will outline its proof.

By adding finitely many points, $X$ can be topologically embedded into a compact Riemann surface $\hat{X}$ so that $\pi$ extends to a holomorphic map $\hat{\pi}: \hat{X} \rightarrow$ $\mathbb{P}_{1}(\mathbb{C})$ (see $[1$, page 82$]$ for details). By $5.0 .10(2), \hat{X}$ is bi-holomorphic with an algebraic curve $\hat{\Gamma}$, hence $X$ is homeomorphic to a co-finite subset $\Gamma$ of $\hat{\Gamma}$.

Obviously $\Gamma$ is not compact, hence $\Gamma$ is a proper subset of $\hat{\Gamma}$. Using RiemannRoch theorem we can find sufficiently many rational functions on $\hat{\Gamma}$ that are regular on $\Gamma$, and embed $\Gamma$ into an affine space. 
Our main goal below is to prove an analogue to Theorem 5.0.11 for definable finite coverings of co-finite subsets of $K$.

5.1. Definable covering maps. We refer to [3] for a definition of a definable space. We always assume that a definable space is definably regular. We use $I$ to denote the closed interval $[0,1]$ in $R$.

Definition 5.1.1. By a definable covering map we mean a definable map $\pi: X \rightarrow$ $B$ between definable spaces that is also a finite covering. A definable $n$-covering map $\pi: X \rightarrow B$ is called trivial if $X$ consists of $n$ definable disjoint sets $X_{1}, \ldots, X_{n}$ and $\pi$ maps each $X_{i}$ homeomorphically onto $B$.

$A$ definable isomorphism of two definable covering maps $\pi: X \rightarrow B$ and $\rho: Y \rightarrow B$ is a definable homeomorphism $f: X \rightarrow Y$ such that $\rho \circ f=\pi$.

Remark 5.1.2. In [5] a definable covering map is defined differently. We will show in Appendix A that in fact both definitions are equivalent. Also, since proofs of some basic properties of definable covering maps are almost identical to analogous statements from Algebraic Topology, we present them in Appendix.

Our goal is to prove the following theorem.

Theorem 5.1.3. maincov Let $B \subseteq K$ be a co-finite subset, $X$ a definably regular definable space, and $\pi: X \rightarrow B$ a definable covering map. Then there exist a nonsingular affine algebraic curve $\Gamma$ over $K$, a regular map $\rho: \Gamma \rightarrow B$ and a definable homeomorphism $f: X \rightarrow \Gamma$ such that $\rho \circ f=\pi$.

To prove the above theorem we will count, up to definable isomorphisms, the number of definable $n$-covering maps of a co-finite $B \subseteq K$ and then, using classical Riemann existence theorem, show that every such covering can be realized by an affine algebraic curve.

We will need the following claim whose proof is given in Appendix.

Claim 5.1.4. contrivial Let $\pi: X \rightarrow B$ be a definable covering map. If $B$ is definably contractible then $\pi$ is trivial.

5.1.1. Definable Paths. Recall that if $X$ is a definable space then a definable path in $X$ is a definable continuous function $\sigma: I \rightarrow X$. If $\sigma: I \rightarrow X$ is a definable path then the point $\sigma(0) \in X$ is called the initial point of $\sigma$, the point $\sigma(1)$ is called the end point of $\sigma$, and we also say that $\sigma$ is a definable path from $\sigma(0)$ to $\sigma(1)$.

A closed path or a loop is a a definable path whose initial point coincides with the end point. If a path $\sigma$ is a loop with the initial point $p$ then we also say that $p$ is the base point of the loop $\sigma$.

Let $\pi: X \rightarrow B$ be a definable covering map and $\sigma: I \rightarrow B$ a definable path. As usual, a definable path $\tau: I \rightarrow X$ is called a lift of $\sigma$ if $\sigma=\pi \circ \tau$.

Claim 5.1.5. onelift Let $\pi: X \rightarrow B$ be a definable covering map, $\sigma: I \rightarrow B$ a definable path and $\tau_{1}, \tau_{2}: I \rightarrow X$ two definable lifts of $\sigma$. If $\tau_{1}(t)=\tau_{2}(t)$ for some $t \in I$ then $\tau_{1}(t)=\tau_{2}(t)$ for all $t \in I$, 
Proof. The set $J=\left\{t \in I: \tau_{1}(t)=\tau_{2}(t)\right\}$ is both open and closed in $I$. Since $I$ is definably connected, $J=I$ or empty.

The proof of the following claim is presented in Appendix.

Claim 5.1.6 (Path lifting). lift Let $\pi: X \rightarrow B$ be a definable finite covering map and $\sigma$ a definable path in $B$ with the initial point $b$. Then for every $p \in \pi^{-1}(b)$ there is a unique definable lift $\tau$ of $\sigma$ with the initial point $p$.

Following [5] we will denote the end point $\tau(1)$ of the above path $\tau$ by $p \star \sigma$.

5.2. K-holomorphic finite cover. In this paper we are interested only in coverings of open subsets of $K$. Let $B$ be an open definable subset of $K$ and $X$ a $K$-manifold. $A K$-holomorphic covering map is a $K$-holomorphic map $\pi: X \rightarrow B$ such that $\pi$ is also a definable covering map.

Claim 5.2.1. cclaim Let $B \subseteq K$ be a definable open set, $X_{1}, X_{2}$ be K-manifolds, and let $\pi_{i}: X_{i} \rightarrow B, i \in\{1,2\}$, be K-holomorphic covering maps. If $f: X_{1} \rightarrow X_{2}$ is a definable isomorphism of definable covering maps then $f$ is $K$-holomorphic.

Proof. Let $\sigma$ be the isomorphism of the two coverings. Since every definable covering map is locally a bijection, we have locally $\sigma=\pi_{1} \pi_{2}^{-1}$ so $\sigma$ is $K$-holomorphic.

5.3. Counting definable covering maps of a punctured plane. We follow ideas from [9].

We fix integers $n, k$ and $k$ distinct points $s_{1}, \ldots, s_{k} \in R^{2}$. Let $S=\left\{s_{1}, \ldots, s_{k}\right\}$ and $B=R^{2} \backslash S$.

We choose a base point $O \in B$ such that for any $s_{i} \in S$ the straight line through $O$ and $s_{i}$ does not contain any other point $s_{j} \in S$.

For every $i=1, \ldots, k$ let $r_{i}$ be the ray in $K$ with endpoint $O$ that passes through $s_{i}$, and let $r_{i}^{+}$denotes the part of $r_{i}$ without the segment $\left[O, s_{i}\right)$, i.e.

$$
r_{i}=\left\{t \cdot \overrightarrow{O s_{i}}: t \in R^{\geqslant 0}\right\} \text { and } r_{i}^{+}=\left\{t \cdot \overrightarrow{O s_{i}}: t \in R^{\geqslant 0}, t \geqslant 1\right\} .
$$

Let $U=R^{2} \backslash \bigcup_{i=1}^{k} r_{i}^{+}$. Obviously $U$ is definably contractible and is contained in $B$.

By Claim 5.1.4, if $\pi: X \rightarrow B$ is a definable $n$-covering map then the restriction of $\pi$ onto $\pi^{-1}(U)$ is trivial. Thus to describe $\pi$ we only need to specify how components of $\pi^{-1}(U)$ are glued along $\pi^{-1}\left(r_{i}^{+} \cap B\right)$. We fix a small positive $\varepsilon \in R$ such for each $s_{i}$ the disk $D_{i}$ of radius $\varepsilon$ centered at $s_{i}$ does not contain neither $O$ nor any other $s_{j} \in S$.

For each $i=1, \ldots, k$ we pick a loop $\sigma_{i}$ in $B$ with base $O$ that starts at $O$ travels towards $s_{i}$ along the ray $r_{i}$ until it reaches the boundary of $D_{i}$, then it travels once counter-clock-wise along the boundary of $D_{i}$, and then comes back to $O$ along $r_{i}$.

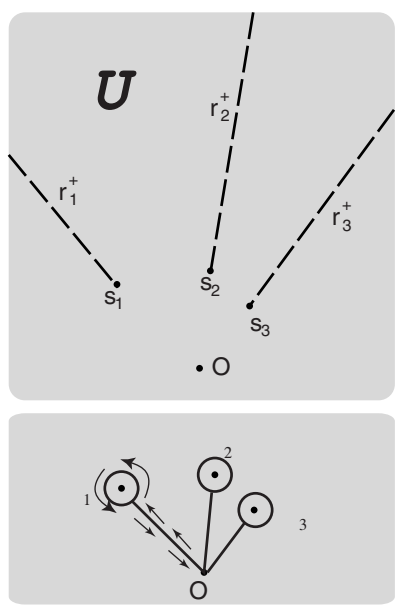


Let $\pi: X \rightarrow B$ be a definable $n$-covering map. For $i=1, \ldots, k$ let $\alpha_{i}$ be the permutation of $\pi^{-1}(O)$ defined as $\alpha_{i}: p \mapsto p * \sigma_{i}$. After an enumeration of $\pi^{-1}(O)$, the sequence $\alpha_{1}, \ldots, \alpha_{k}$ will correspond to a tuple $\left(g_{1}, \ldots, g_{k}\right) \in \operatorname{Sym}(n)^{k}$. We will denote by $\left\lfloor g_{1}, \ldots, g_{k}\right\rfloor$ the conjugacy class of $\left(g_{1}, \ldots, g_{k}\right)$, namely

$$
\left\lfloor g_{1}, \ldots, g_{k}\right\rfloor=\left\{\left(h g_{1} h^{-1}, \ldots, h g_{k} h^{-1}\right): h \in \operatorname{Sym}(n)\right\}
$$

Obviously, the tuple $\left(g_{1}, \ldots, g_{k}\right)$ depends on an enumeration of $\pi^{-1}(O)$, but its conjugacy class does not.

We call the conjugacy class $\left\lfloor g_{1}, \ldots, g_{k}\right\rfloor$ the monodromy data of the covering $\pi: X \rightarrow B$ (with the base point $O$ ).

Theorem 5.3.1. mono For every tuple $\left(g_{1}, \ldots, g_{k}\right) \in \operatorname{Sym}(n)^{k}$, up-to a definable isomorphism, there is a unique definable $n$-covering $\pi: X \rightarrow B$ with the monodromy data $\left\lfloor g_{1}, \ldots, g_{k}\right\rfloor$.

Proof. For existence we will actually show that every monodromy data can be realized by a semi-algebraic covering.

We choose pairwise disjoint tubular neighborhoods $V_{i}$ of $r_{i}^{+}$in $B$. We denote by $V_{i}^{l}$ and $V_{i}^{r}$ the left and the right connected components of $V_{i} \cap U$.

Let $X_{1}=U \times\{1, \ldots, n\}, X_{2}=\bigcup_{i=1}^{k} V_{i} \times\{1, \ldots, n\}$, and $A=\bigcup_{i=1}^{k}\left(V_{i}^{l} \cup V_{i}^{r}\right) \times\{1, \ldots, n\}$. We consider $A$ as a subset of $X_{1}$. Let $f: A \rightarrow X_{2}$ be the map defined as

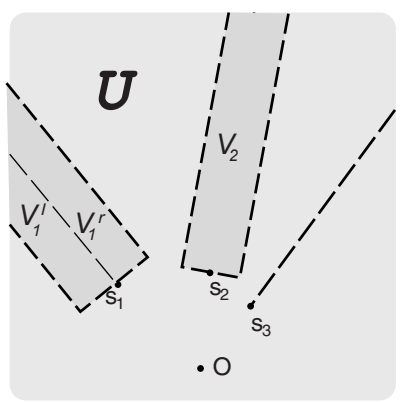

$$
f((p, j))= \begin{cases}(p, j) & \text { if } p \in V_{i}^{r} \\ \left(p, g_{i}(j)\right) & \text { if } p \in V_{i}^{l} .\end{cases}
$$

Let $X=X_{1} \coprod_{f} X_{2}$ be the space obtained by attaching $X_{1}$ to $X_{2}$ via $f$ (see [3, Chapter 10, Section 2.9] for a precise definition), and $\pi: X \rightarrow B$ be the map $(p, j) \mapsto p$. It is easy to see that $\pi$ is a definable $n$-covering map with the monodromy data $\left\lfloor g_{1}, \ldots, g_{k}\right\rfloor$.

For a uniqueness, let $\tau: Y \rightarrow B$ be a definable $n$-covering with the monodromy data $\left\lfloor g_{1}, \ldots, g_{k}\right\rfloor$.

We can choose an enumeration of $\pi^{-1}(O)$ so that, with respect to this enumeration, $\alpha_{i}=g_{i}$ for $i=1, \ldots, k$. It is not hard to see that the covering $\tau: Y \rightarrow B$ is definably isomorphic to the semialgebraic covering $\pi: X \rightarrow B$ constructed above.

Corollary 5.3.2. main 1 For any integers $k, n \in \mathbb{N}^{>0}$ there is a number $N(k, n)$ such that for any set $S \subseteq R^{2}$ with $|S|=k$ up to a definable isomorphism there are exactly $N(k, n)$ definable $n$-covering maps $\pi: X \rightarrow R^{2} \backslash S$. This number $N(k, n)$ depends on $n$ and $k$ only and does not depend on neither the structure $\mathcal{R}$, nor the set $S$. 
Proof. It follows from the previous discussion that the number $N(k, n)$ is equal to the number of conjugacy classes in $\operatorname{Sym}(n)^{k}$.

5.3.1. The classical case. class In the case, when the field $R$ is the field of the real number, it is not hard to see that two definable $n$-coverings of a co-finite subset of $\mathbb{R}^{2}$ are definably isomorphic if and only if they are isomorphic topologically.

Indeed, let $\pi_{i}: X_{i} \rightarrow B, i=1,2$, be definable $n$-coverings of a co-finite subset $B$ of $\mathbb{R}^{2}$. If $f: X_{1} \rightarrow X_{2}$ is a homeomorphism, then, since in this case Claim 5.1.4 and Claim 5.1.5 hold without definability assumptions (e.g. see [7]), $\pi_{1}$ and $\pi_{2}$ have the same monodromy data, hence definably isomorphic.

\subsection{Proof of Theorem 5.1.3. We need the following claim.}

Claim 5.4.1. algcov1 Let $n \in \mathbb{N}, B \subseteq K$ a co-finite subset, $\Gamma$ a non-singular affine algebraic curve over $K$, and $\pi$ a regular function on $\Gamma$. Then $\pi: \Gamma \rightarrow B$ is a definable $n$-covering (with respect to the induced $R$-topology) if and only if $\pi$ is surjective and the size of $\pi^{-1}(y)$ is exactly $n$ for every $y \in B$.

Proof. By Claim 3.1.1, we need only to check that $\pi$ is a local homeomorphism. Since $\Gamma$ is non. Let $\Gamma_{1}, \ldots, \Gamma_{l}$ be the irreducible components of $\Gamma$, and let $\pi_{i}=\pi \mid \Gamma_{i}$.

For $i=1, \ldots, l$ and $y \in B$ we have that $\left|\pi^{-1}(y)\right| \leqslant \operatorname{deg}\left(\pi_{i}\right)$ with equality for almost all $y \in B$. Thus the sum of degrees of $\pi_{i}$ is $n$, and $\left|\pi_{i}^{-1}(y)\right|=\operatorname{deg}\left(\pi_{i}\right)$ for all $y \in B$.

Thus every $\pi_{i}$ is an unramified finite map, its differential does not vanish anywhere on $\Gamma_{i}$, (see [19, Chapter II, Section 6.3, Theorem 5]), hence it is a local diffeomorphism

The above claim allows us to give a purely algebraic definition of an algebraic $n$-covering. Namely, let $B \subseteq K$ be a co-finite subset. A pair $\langle\Gamma, \pi\rangle$ is an algebraic $n$-covering of $B$ if $\Gamma$ is a non-singular affine algebraic curve over $K, \pi$ is a regular function on $\Gamma, B$ is the range of $\pi$, and $\left|\pi^{-1}(y)\right|=n$ for all $y \in B$. Two algebraic $n$-coverings $\langle\Gamma, \pi\rangle$ and $\left\langle\Gamma^{\prime}, \pi\right\rangle$ are isomorphic if there is a regular map $f: \Gamma \rightarrow \Gamma^{\prime}$ such that $\pi=\pi^{\prime} \circ f$. If $L$ is a subfield of $K$ and both $\Gamma$ and $\pi$ are defined over $L$ then we say that $\langle\Gamma, \pi\rangle$ is a covering over $L$.

Theorem 5.4.2. main1.5 Let $\langle\Gamma, \pi\rangle$ and $\left\langle\Gamma^{\prime}, \pi^{\prime}\right\rangle$ be two algebraic $n$-coverings of a co-finite set $B \subset K,|K \backslash B|=k$. Then they are isomorphic as algebraic covers if and only if they are isomorphic as definable covering maps.

In particular, there are at most $N(k, n)$ (see 5.3.2) algebraic $n$-coverings of $B$, independently of $K$ and of $B$.

Proof. Clearly, every algebraic isomorphism of the two covers is also definable, so we need to prove the converse.

Assume that $\sigma: \Gamma \rightarrow \Gamma^{\prime}$ is a definable isomorphism. Because $\Gamma \subset K^{n}$ and $\Gamma^{\prime} \subset K^{m}$ are non-singular curves, they are $K$-manifolds. By Claim 5.2.1, the map $\sigma$ is $K$-holomorphic, hence by our version of Chow's Theorem (Theorem 2.3.2), it is an algebraic map. The final clause follows from 5.3.2. 
Thus, in order to prove Theorem 5.1.3 it is sufficient to prove the following purely algebraic result.

Theorem 5.4.3. algcov Let $L$ be an algebraically closed field characteristic zero, $B \subseteq L$ a co-finite subset, $k=|L \backslash B|$. Then there are exactly $N(k, n)$ nonisomorphic algebraic $n$-coverings of $B$ defined over the field $L$.

Proof. We fix integers $n$ and $k$.

For an algebraically closed characteristic zero field $L$ and a co-finite subset $B \subseteq L$ with $|L \backslash B|=k$ we will denote by $N^{\prime}(L, B)$ the number of nonisomorphic algebraic $n$-coverings of $B$ defined over the field $L$. We need to prove that $N^{\prime}(L, B)=N(k, n)$.

Remark 5.4.4. Using the equivalence of categories of algebraic finite coverings and ramified extensions of $L(X)$, this result can be derived from the main result of [4]. Here we present an alternative proof.

It follows from Theorem 5.4.2 that $N^{\prime}(L, B) \leqslant N(k, n)$, and we only need to show that $N^{\prime}(L, B) \geqslant N(k, n)$.

First notice that the above inequality holds in the case $L=\mathbb{C}$. Indeed, by Corollary 5.3.2 (applied to the o-minimal real field), and discussion in Section 5.3.1, there are $N(k, n)$-many different topological $n$-coverings of $B \subseteq \mathbb{C}$. By Theorem 5.0.11 (i.e., by the classical Riemann existence Theorem), every such covering is topologically isomorphic to an $n$-algebraic covering of $B$. We therefore have $N(k, n)$-many algebraic $n$-coverings of $B$ that are non-isomorphic as algebraic curves (since they are not even topologically isomorphic).

For the purpose of getting a contradiction we assume that for some algebraically closed characteristic zero field $L$ and $B \subseteq L$ with $|L \backslash B|=k$ such that $N^{\prime}(L, B)<N(k, n)$. Let $N^{\prime}=N^{\prime}(L, B)$.

Let $\left\langle\Gamma_{1}, \pi_{1}\right\rangle, \ldots,\left\langle\Gamma_{N^{\prime}}, \pi_{N^{\prime}}\right\rangle$ be non-isomorphic algebraic coverings of $B$ defined over $L$. Thus if $\langle\Gamma, \pi\rangle$ is an algebraic covering of $B$ defined over $L$ then it is isomorphic to one of the $\left\langle\Gamma_{i}, \pi_{i}\right\rangle^{\prime}$ s. Let $S=L \backslash B$. By restricting ourselves to a subfield of $L$ over which $S$ and $\Gamma_{i}, \pi_{i}$ are all defined we may assume that $L$ is a countable field.

Since the field $\mathbb{C}$ is saturated, we can embed $L$ into $\mathbb{C}$ and assume that $L$ is a countable subfield of $\mathbb{C}$. Let $\widetilde{\Gamma}_{i}$ and $\widetilde{\pi}_{i}$ be $\mathbb{C}$-points of $\Gamma_{i}$ and $\pi_{i}$ respectively; and let $\widetilde{B}=\mathbb{C} \backslash S$

Since $N^{\prime}<N(k, n)$, it follows from the case $L=\mathbb{C}$ that there exists an algebraic $n$-covering $\langle\mathcal{C}, \rho\rangle$ of $\tilde{B}$ that is not isomorphic over $\mathbb{C}$ to any of the $\left\langle\widetilde{\Gamma}_{i}, \widetilde{\pi}_{i}\right\rangle^{\prime}$ s. As we will show, this cover is isomorphic to a cover over $L$ and this leads to a contradiction.

We choose polynomials $p_{1}(\bar{x}, \bar{u}), \ldots, p_{m}(\bar{x}, \bar{u}), q(\bar{x}, \bar{u})$ over $\mathbb{Z}$, and $\bar{d} \in \mathbb{C}^{l}$ such that $\mathcal{C}=\left\{\bar{z} \in \mathbb{C}^{s}: p_{1}(\bar{z}, \bar{d})=\cdots=p_{m}(\bar{z}, \bar{d})=0\right\}$ and $\rho=q(\bar{x}, \bar{d})$.

For $\bar{a} \in \mathbb{C}^{l}$ let $\mathcal{C}_{\bar{a}}=\left\{\bar{z} \in \mathbb{C}^{s}: p_{1}(\bar{z}, \bar{a})=\cdots=p_{m}(\bar{z}, \bar{a})=0\right\}$ and $\rho_{\bar{a}}=q(\bar{x}, \bar{a})$. 
It is easy to see that the set

$$
A=\left\{\bar{a} \in \mathbb{C}^{l}:\left\langle\mathcal{C}_{\bar{a}}, \rho_{\bar{a}}\right\rangle \text { is an algebraic } n \text {-covering of } \widetilde{B}\right\}
$$

is definable (in the language of rings) over the field $L$.

Consider the equivalence relation $\mathcal{E}$ on $A$ given as

$\mathcal{E}\left(\bar{a}, \bar{a}^{\prime}\right)$ iff

the algebraic coverings $\left\langle\mathcal{C}_{\bar{a}}, \rho_{\bar{a}}\right\rangle$ and $\left\langle\mathcal{C}_{\bar{a}^{\prime}}, \rho_{\bar{a}^{\prime}}\right\rangle$ are isomorphic.

It is not hard to see that $\mathcal{E}\left(\bar{x}, \bar{x}^{\prime}\right)$ is $\bigvee$-definable over $L$, i.e. it is a countable union of sets definable over $L$.

By $5.4 .2, \mathcal{E}$ has at most $N(k, n)$-many classes. Since $\mathbb{C}$ is $\aleph_{1}$-saturated, every $\bigvee$-definable equivalence relation on $A$ with finitely many classes must be definable (every $\mathcal{E}$-class is $\bigvee$-definable with $\bigvee$-definable complement, hence definable). Since $L$ is elementary substructure of $\mathbb{C}$, and $\mathcal{E}$ is a definable (over $L$ ) equivalence relation with finitely many classes, every $\mathcal{E}$-class must have a representative in $L$. Thus there is $\bar{d}^{\prime} \in L^{l}$ with $\mathcal{E}\left(\bar{d}, \bar{d}^{\prime}\right)$. The algebraic covering $\left\langle\mathcal{C}_{\bar{d}^{\prime}}, \rho_{\bar{d}^{\prime}}\right\rangle$ is defined over $L$ and is not isomorphic to any of the $\left\langle\Gamma_{i}, \pi_{i}\right\rangle^{\prime}$ s. A contradiction.

This ends the proof of Theorem 5.1.3.

\section{Proof of the MAIN Theorem}

In this section we will prove the main theorem stated in the introduction.

Theorem 6.0.5. main Let $M$ be a strongly minimal mild manifold. Then either the structure $\mathcal{A}(M)$ is locally modular or $M$ is $K$-biholomorphic to a non-singular algebraic curve over $K$.

Proof. Assume $\mathcal{A}(M)$ is not locally modular. Then, by Theorem 4.3 .3 , there is a non-constant $K$-meromorphic $\pi: M \rightarrow K$. By Claim 4.3.6, there is a finite subset $Z \subseteq M$ and a finite subset $S \subseteq K$ such that $\pi: M \backslash Z \rightarrow K \backslash S$ is a $K$-holomorphic finite covering. Using Theorem 5.1 .3 we can find a non-singular affine curve $\Gamma$ and a $K$-biholomorphism $g: M \backslash Z \rightarrow \Gamma$.

Since $M$ is definably connected and $Z$ is finite, $M \backslash Z$ is definably connected, hence $\Gamma$ is irreducible.

Taking a projectiviztion of $\Gamma$ and then, if needed, a normalization, we can find a non-singular projective curve $\mathcal{C}$ and an injective regular map $h: \Gamma \rightarrow C$ whose range is co-finite in $\mathcal{C}$. Composing $g$ with $h$ we obtain a $K$-holomorphic injective map $f: M \backslash Z \rightarrow \mathcal{C}$ whose range is co-finite in $\mathcal{C}$.

We first claim that $f$ can be extended to a $K$-holomorphic map $F: M \rightarrow \mathcal{C}$.

Notice that $\operatorname{dim}_{K} M=1$ and $\mathcal{C}$ is definably compact (as a definable closed subset of a projective space). Let $G_{f} \subseteq M \times \mathcal{C}$ be the graph of $f$ and $A$ its topological closure. By [14, Corollary 9.1], $A$ is a $K$-analytic subset of $M \times \mathcal{C}$. Since the o-minimal dimension of $G_{f}$ is 2 we have that o-minimal dimension of $A$ is also 2 . 
Since all proper $k$-analytic subsets of $\mathcal{C}$ are finite, for every $m \in M$ the set $A_{m}=A \cap(\{m\} \times \mathcal{C})$ is either finite or co-finite. Because the o-minimal dimension of $A \backslash G_{f}$ is at most one, for every $z \in Z$, the set $A_{z}$ must be finite.

Pick $z \in Z$. Let $U \subseteq M$ be a definable open subset of $M$ definably $K$ biholomorphic to an open disk $D \subseteq K$ with $U \cap Z=\{z\}$. By [12, Theorem 2.37 ], it is sufficient to show that $f$ can be extended continuously to $z$. Since $\mathcal{C}$ is definably compact, $f(\gamma(t))$ has a limit in $\mathcal{C}$ for every definable curve $\gamma(t)$ in $M$ approaching $z$, hence $A_{z}$ is not empty. Since $U \backslash\{z\}$ is definably connected, $A_{z}$ is also definably connected (see [12, Fact 2.2]), hence it contains exactly one point. Using the definable compactness of $\mathcal{C}$ it is not hard to see that $A$ is the graph of a continuous function $F: M \rightarrow \mathcal{C}$ extending $f$.

We claim that $F$ is injective. Indeed if $F(a)=F(b)$ for some $a \neq b \in M$, then, by Open Mapping Theorem [12, Corollary 2.34], for every $a^{\prime} \in M$ close enough to $a$ there is $b^{\prime} \in M$ close enough to $b$ such that $F\left(a^{\prime}\right)=F\left(b^{\prime}\right)$. This contradicts to the injectivity of $f$.

Let $\mathcal{C}^{\prime}$ be the range of $F$. Then $\mathcal{C}^{\prime}$ is a quasi-projective smooth curve $K$ biholomorphic to $M$.

Example 6.0.6. As was shown in [15], there are one-dimensional definably compact $K$-groups (these are $K$-manifolds with $K$-holomorphic group operations) which are not isomorphic as $K$-manifolds to any algebraic curve. It follows from Theorem 6.0.5 that the every such $K$-group, with all its induced $K$-analytic structure, is locally modular.

\section{Appendix A. More on Definable finite covers}

app

Proof of Claim 5.1.6. Let $\pi: X \rightarrow B$ be a definable $n$-covering map, $\sigma: I \rightarrow B$ a definable path with the initial point $b$, and $p \in \pi^{-1}(b)$.

Uniqueness follows from Claim 5.1.5, and we only need to show existence of a lift $\tau: I \rightarrow X$ of $\sigma$ with the initial point $p$.

Using definable choice we can find $n$ definable functions $g_{1} \ldots, g_{n}: B \rightarrow X$ such that $\pi^{-1}(z)=\left\{g_{1}(z), \ldots, g_{n}(z)\right\}$ for all $z \in B$. Using basic properties of o-minimal structures we can find $0=t_{0}<t_{1}<\ldots<t_{k}=1$ such that for every $j=1, \ldots, n$ and $i=1, \ldots, k$ the function $h_{j}(t)=g_{j}(\sigma(t))$ is continuous on $\left(t_{i-1}, t_{i}\right)$. By induction on $i=0, \ldots, k$ we construct a definable continuous function $\tau_{i}:\left[0, t_{i}\right] \rightarrow X$ such that $\tau_{i}(0)=p$ and $\pi \circ \tau_{i}=\sigma$ on $\left[0, t_{i}\right]$. We set $\tau_{0}(0)=p$. Assume we have $\tau_{i}$. Let $V$ be a definable open neigbourhood of $\tau_{i}\left(t_{i}\right)$ such that $\pi$ maps $V$ homeomorphically onto an open neighborhood $U$ of $\sigma\left(t_{i}\right)$. It is not hard to see that there is unique $j \in\{1, \ldots, n\}$ such that $g_{j}(\sigma(t)) \in V$ for all $t \in\left(t_{i}, t_{i}+\varepsilon\right)$ for some sufficiently small $\varepsilon>0$. Let

$$
\tau_{i+1}^{\prime}= \begin{cases}\tau_{i}(t) & \text { if } t \in\left[0, t_{i}\right) \\ g_{j}(\sigma(t)) & \text { if } t \in\left(t_{i}, t_{i+1}\right) .\end{cases}
$$


Obviously $\tau_{i+1}^{\prime}$ is continuous on $\left[0, t_{i+1}\right)$ and $\pi \circ \tau_{i+1}^{\prime}=\sigma$ on $\left[0, t_{i+1}\right)$. We now set $\tau_{i+1}=\tau_{i+1}^{\prime}$ on $\left[0, t_{i+1}\right)$ and $\tau_{i+1}\left(t_{i+1}\right)=q$, where $q$ is a unique point in $\pi^{-1}\left(t_{i+1}\right)$ with $\lim _{t \rightarrow t_{i+1}^{-}} \tau_{i+1}^{\prime}(t)=q$.

Remark A.0.7. It is not hard to see that the above prove can be generalized to a definable family of definable paths. Namely if $\sigma_{a}, a \in A$, is a definable family of definable paths in $B$, and $f: A \rightarrow X$ is a definable function with $\pi(f(a))=\sigma_{a}(0)$ then there is a definable family of paths $\tau_{a}: I \rightarrow X, a \in A$, such that each $\tau_{a}$ is a lift of $\sigma_{a}$ with $\tau_{a}(0)=f(a)$.

Proof of Claim 5.1.4. Let $\pi: X \rightarrow B$ be a definable $n$-covering map with $B$ definably contractible, i.e. there is $b \in B$ and a definable continuous $F: B \times I \rightarrow B$ such that

$$
F(z, 1)=z \text { and } F(z, 0)=b \text { for all } z \in B .
$$

We need to show that $\pi$ is a trivial cover. Recall that a definable section of $\pi$ is a definable continuous map $\rho: B \rightarrow X$ such that $\pi \circ \rho=\mathrm{id}_{B}$. If $\rho$ is a definable section of $\pi$ then $\pi$ restricted to $X \backslash \rho(B)$ is a definable $n$-1-covering map. Therefore, applying an induction on $n$, it is sufficient to show that there is a definable section of $\pi$.

We fix $p \in \pi^{-1}(b)$. For $z \in B$ let $\sigma_{z}$ be the definable path $t \mapsto F(z, t)$. Thus we have a definable family $\sigma_{z}, z \in B$, of definable paths in $B$ all with the same initial point $b$. By the previous remark, there is a definable family $\tau_{z}, z \in B$, of definable paths in $X$ all with the initial point $p$ such that $\pi\left(\tau_{z}(t)\right)=F(z, t)$ for all $z \in B, t \in I$. Let $\widehat{F}(z, t): B \times I \rightarrow Z$ be the map $F(z, t)=\tau_{z}(t)$. We have $\pi \circ \tilde{F}=F, \widehat{F}(z, 0)=p$ for all $z \in B$, and for each $z \in B$ the map $t \mapsto \widehat{F}(z, t)$ is continuous on $I$.

Let $\rho: B \rightarrow X$ be the map $z \mapsto \widehat{F}(z, 1)$. Clearly $\pi \circ \rho=\operatorname{id}_{B}$, and it remains to show that $\rho$ is continuous.

Let $z_{0} \in B$ and $x_{0}=\rho\left(z_{0}\right)$. We will show that $\rho$ is continuous at $z_{0}$.

Let $\sigma=\sigma_{z_{0}}$, and $\tau_{1}, \ldots, \tau_{n}: I \rightarrow X$ be all lifts of $\sigma$ with $\tau_{1}=\tau_{z_{0}}$. Thus $\sigma$ is a path in $B$ from $b$ to $z_{0}$, and $\tau_{1}$ is a path in $X$ from $p$ to $x_{0}$. We will denote by $\widetilde{\sigma}$ the range of $\sigma$ and by $\widetilde{\tau}_{i}$ the range of $\tau_{i}$.

Since $X$ is definably normal space, and, by Claim 5.1 .5 , the sets $\widetilde{\tau}_{i}$ are pairwise disjoint, there are definable pair-wise disjoint open subsets $V_{i} \subseteq X, i=$ $1, \ldots, n$, with $\widetilde{\tau}_{i} \subseteq V_{i}$. Since $\pi$ is a local homeomorphism, the set $W=\cap_{i=1}^{n} \pi\left(V_{i}\right)$ is open in $B$, and it contains $\widetilde{\sigma}$. Let $V=\pi^{-1}(W) \cap V_{1}$. Obviously $V \subseteq X$ is definable open, it contains $\widetilde{\tau}_{1}$, and $\pi$ maps $V$ homeomorphically onto $W$.

We claim that

$(\star)$ There is an open definable subset $O \subseteq B$ containing $z_{0}$ such that $W$ contains the range of $O \times I$ under $F$.

Let's show that $(\star)$ is sufficient. Indeed, if $z \in O$ then the range of $\sigma_{z}$ is in $W$, the range of $\tau_{z}$ is in $V$, hence $\rho(z)$ is the unique element $x$ in $V$ with $\pi(x)=z$. Thus $\rho$ maps $O$ homeomorphically onto $\rho(O)$ and is continuous at $z_{0}$. 
We are left to prove $(\star)$. Since by [3, Chapter 10, Theorem 1.8], all definable regular spaces are affine, we can assume that $X \subseteq R^{m}$. Let $d(\cdot, \cdot): R^{m} \times R^{m} \rightarrow R$ be the distance function on $R^{m}$.

For $t \in I$ let

$$
h(t)=\sup \left\{\varepsilon \in R: F(z, t) \in W \text { for all } z \in B \text { with } d\left(z_{0}, z\right)<\varepsilon\right\} .
$$

Since $F$ is continuous and $F\left(z_{0}, t\right) \in W$ for all $t \in I$, it is not hard to see that $h(t)>0$ for all $t \in I$, and also for every $t \in I$ there is $\varepsilon>0$ such that $h\left(t^{\prime}\right)>\varepsilon$ for $t^{\prime} \in I$ sufficiently close to $t$. Since $I$ is definably compact, using Curve Selection, we obtain that there is $\varepsilon_{0}>0$ such that $h(t)>\varepsilon_{0}$ for all $t \in I$. We can take $O=\left\{z \in B: d\left(z, z_{0}\right)<\varepsilon_{0}\right\}$.

The following corollary shows that our definition of covering maps is equivalent to the definition in [5]

Corollary A.0.8. Let $\pi: X \rightarrow B$ be a definable $n$-covering map. Then $B$ can be covered by finitely many open definable sets $V_{i}, i=1, \ldots, k$ such that for each $\pi^{-1}\left(V_{i}\right)$ consists of $n$ pair-wise disjoint definable sets $U_{i}^{1}, \ldots, U_{i}^{n}$ and $\pi$ maps each $U_{i}^{j}$ homeomorphically onto $V_{i}$.

Proof. It is sufficient to show that $B$ can be covered by finitely many open definable definably contractible sets $V_{i}$. We can take $V_{i}$ to be star neighborhoods of vertexes in the barycentric subdivision of any triangulation of $X$.

\section{REFERENCES}

[1] Jean-Benoît Bost, Introduction to compact Riemann surfaces, Jacobians, and abelian varieties, From number theory to physics (Les Houches, 1989), Springer, Berlin, 1992, pp. 64-211.

[2] Claude Chevalley, Introduction to the theory of algebraic functions of one variable, Mathematical Surveys, No. VI, American Mathematical Society, Providence, R.I., 1963.

[3] Lou van den Dries, Tame topology and o-minimal structures, London Mathematical Society Lecture Note Series, vol. 248, Cambridge University Press, Cambridge, 1998.

[4] Lou van den Dries and Paulo Ribenboim, Application de la théorie des modèles aux groupes de Galois de corps de fonctions, C. R. Acad. Sci. Paris Sér. A-B 288 (1979), no. 17, A789-A792 (French, with English summary).

[5] Mário J. Edmundo and Margarita Otero, Definably compact abelian groups, J. Math. Log. 4 (2004), no. 2, 163-180.

[6] Robin Hartshorne, Algebraic geometry, Springer-Verlag, New York, 1977. Graduate Texts in Mathematics, No. 52.

[7] Allen Hatcher, Algebraic topology, Cambridge University Press, Cambridge, 2002.

[8] Ehud Hrushovski and Boris Zilber, Zariski geometries, J. Amer. Math. Soc. 9 (1996), no. 1, 1-56. 
[9] A. Hurwitz, Ueber Riemann'sche Flächen mit gegebenen Verzweigungspunkten, Math. Ann. 39 (1891), no. 1, 1-60 (German).

[10] Rahim Moosa, A nonstandard Riemann existence theorem, Trans. Amer. Math. Soc. 356 (2004), no. 5, 1781-1797 (electronic).

[11] R. Moosa and S. Starchenko, $K$-analytic versus ccm-analytic sets in nonstandard compact manifolds, Fund. Math.

[12] Ya'acov Peterzil and Sergei Starchenko, Expansions of algebraically closed fields in o-minimal structures, Selecta Math. (N.S.) 7 (2001), no. 3, 409-445.

[13] _ Expansions of algebraically closed fields. II. Functions of several variables, J. Math. Log., to appear.

[14] _ Complex analytic geometry in a nonstandard setting, Model Theory with applications to algebra and analysis, Vol. I, Cambridge University Press, Cambridge, 2008.

[15] _ Uniform definability of the Weierstrass $\wp$ functions and generalized tori of dimension one, Selecta Math. (N.S.) 10 (2004), no. 4, 525-550.

[16] _ Complex analytic geometry and analytic-geometric categories, J. Reine Angew. Math., to appear.

[17] Anand Pillay, Some model theory of compact complex spaces, geometry (Ghent, 1999), Contemp. Math., vol. 270, Amer. Math. Soc., Providence, RI, 2000, pp. 323-338.

[18] Anand Pillay and Martin Ziegler, Jet spaces of varieties over differential and difference fields, Selecta Math. (N.S.) 9 (2003), no. 4, 579-599.

[19] Igor R. Shafarevich, Basic algebraic geometry. 1, 2nd ed., Springer-Verlag, Berlin, 1994. Varieties in projective space; Translated from the 1988 Russian edition and with notes by Miles Reid.

[20] B. Zil'ber, Model theory and algebraic geometry, Proceedings of the 10th Easter Conference on Model Theory, Seminarberichte, vol. 93, Humboldt Univ. Berlin, 1993, pp. 202-222.

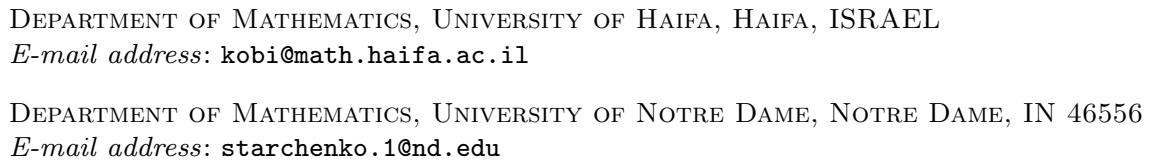

